\title{
REACTOR NOISE EXPERIMENTS CONSIDERING HIGH FREQUENCIES IN THE IPEN/MB-01 REACTOR
}

\author{
Adimir dos Santos and Diogo Feliciano dos Santos \\ Instituto de Pesquisas Energéticas e Nucleares \\ Av. Prof. Lineu Prestes, 2242 CEP 05508000 São Paulo, SP, Brasil \\ adsantos@usp.br,diogofs@msn.com
}

\begin{abstract}
Reactor noise experiments at high frequencies (up to $100 \mathrm{kHz}$ ) in subcritical configurations have been performed at the IPEN/MB-01 research reactor facility. The core configuration considered a short version of the IPEN/MB-01 core in a $24 \times 26$ rectangular array of fuel rods. The subcritical configurations considered the control banks totally withdrawn and the moderator poisoned with $\mathrm{H}_{3} \mathrm{BO}_{3}$. The boron concentrations were: $286.8 \pm 10$ and $578.6 \pm 10 \mathrm{ppm}$. The pulses of two ${ }^{3} \mathrm{He}$ detectors in the reflector region were summed and inserted into the IPEN/MB-01 Correlator and the APSD (Auto Power Spectral Density) was inferred through a mathematical model. The analysis reveals that the APSD in this frequency range is best described by a four-mode decay model. According to the two-region two-group (thermal and fast) kinetic model developed in this work, the first two decay modes describe the thermal group and the other two describe the fast group. After a tedious and severe analyses of the least-square fit of the experimental data, it was concluded that the kinetic behavior of the thermal and fast neutrons can be considered uncoupled. The analysis of the experimental data is still in progress and only the thermal group of the case of $286.8 \mathrm{ppm}$ of boron was analyzed and some parameters could be inferred. The most important one so far is the prompt neutron generation time in the core region that could be inferred with a good level of accuracy.
\end{abstract}

KEYWORDS: Reactor Noise, APSD Measurements, Reflected Core Model, Neutron Generation Time, Two-Group kinetic Model

\section{INTRODUCTION}

Reactor noise applications in research reactors have enjoyed a long history in the reactor physics field $[1,2]$. Most of the applications consider the determination of power, $\beta_{\text {eff, }}$ and subcritical reactivities. However, a common characteristic of these applications is that they rely on the validity of the point kinetic model and a single decay mode ( $\alpha$-mode) which is not applicable in all situations. Beyond that, the noise measurements are performed up to $10.0 \mathrm{kHz}$. Several studies performed at the IPEN/MB-01 research reactor facility indicate that its core is more appropriately described by a two-region model as proposed by Spriggs [3]. Particularly, several parameters such as $\beta_{\text {eff }}$ [4] and subcritical reactivities [5] were measured employing this two-region model.

The purposes of this work are: a) to extend the measurement of the APSD (Auto Power Spectral Density) to frequencies up to $100 \mathrm{kHz}, \mathrm{b}$ ) to developed a two-region two-group kinetic model to interpret the measured data, and c) to analyze the kinetics of the thermal neutrons in the reactor system for the 286.8 
ppm of natural boron case. The experimental data can be very useful to infer important quantities of the interest to the reactor physics field such as subcritical reactivity, neutron lifetimes and generation times in the core and in the reflector regions. Two subcritical cases with distinct boric acid concentrations (286.8 and $578.6 \mathrm{ppm}$ of natural boron) were considered in this work.

The core configuration considered a short version of the IPEN/MB-01 core in a $24 \times 26$ rectangular array of fuel rods, as shown in Fig. 1. The subcritical system was driven by the Am-Be start-up source $(1.0 \mathrm{Ci})$ of the facility. Two ${ }^{3} \mathrm{He}$ Centronic detectors as shown in Fig. 1 were placed symmetrically in the reflector region to get the neutron counts for the IPEN/MB-01 Correlator. The counts were then summed in order to get a better statistic in the measurements. A complete description of the IPEN/MB-01 core can be found elsewhere [6].

\section{THE MEASUREMENT APPROACH}

The data acquisition and processing system employ the following procedure shown in Figure 1.

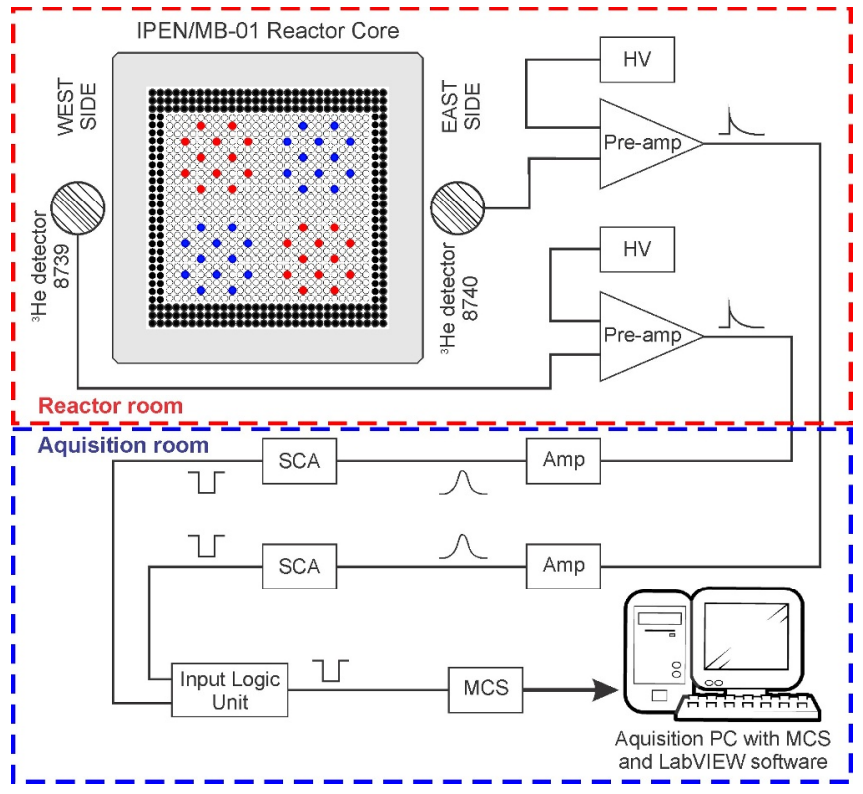

Figure 1. The IPEN/MB-01 configuration and the APSD Measurement System.

Neutron pulses from the detectors are formatted and amplified by preamplifiers and amplifiers.

Subsequently, they were discriminated from the $\gamma$-radiation through the Lower Level Discriminator of the single channel module (Single-Channel Analyzer - SCA). Negative logical pulses are generated in the output of the single channel (standard NIM fast negative) with $25 \mathrm{~ns}$ width and $-5 \mathrm{~V}$ of amplitude on $50 \Omega$ impedance. Since the subcriticality level is too low for the two born diluted cases considered here, the negative logic pulses were summed in a logic OR (logic $X=A+B$ ) employing an Input Logic Unit. A multichannel scaler (MCS) board registers the time intervals between a trigger signal and the subsequent logical pulses. The dwell time provides the maximum frequency to be analyzed, and the number of channels gives the corresponding frequency resolution. The data acquisition control was performed with Virtual Instruments (VI's) specially developed at the IPEN. These VI's were written in a Graphical programming language employing the software LabVIEW 5.1 (Laboratory Virtual Instrument Engineering Workbench). The APSD is generated by means of Fast Fourier Transform (FFT). This whole 
system is called IPEN/MB-01 Correlator. This procedure is totally analogous to Kitamura's work [7]. The experiment set up considers the dwell time equal to $5 \mu \mathrm{s}$, which results in a maximum frequency of $100 \mathrm{kHz}$ (single-sided spectrum), and the number of channels, in the time domain, was set to be 62500 (double sided), which results in a resolution of $3.2 \mathrm{~Hz}$ in the frequency domain. Each experimental point of the APSD has an error bar given by $1 / \sqrt{N}(\%)$, where $N$ is the number of averages [8].

The APSD measurements performed by the Correlator considered 3000 averages in each acquisition. The graphic processing time for each acquisition was 35 minutes. The APSD was very sensitive to the electronic setup; mainly to the amplifier shaping time. A value of $2 \mathrm{~Hz}$ was found adequate for this variable. The average count rates (cps) were $923 \pm 11$ and $198.2 \pm 1.1 \mathrm{cps}$ for the 286.8 and $578.6 \mathrm{ppm}$ of natural boron cases, respectively. The number of acquisitions was 12 and 174, respectively, for the 286.8 ppm and $578.6 \mathrm{ppm}$ cases. The number of acquisitions for the $578.6 \mathrm{ppm}$ case was higher due to its high degree of subcriticality. The measured APSDs for the 286.8 and $578.6 \mathrm{ppm}$ cases of natural boron are shown in Fig. 2.
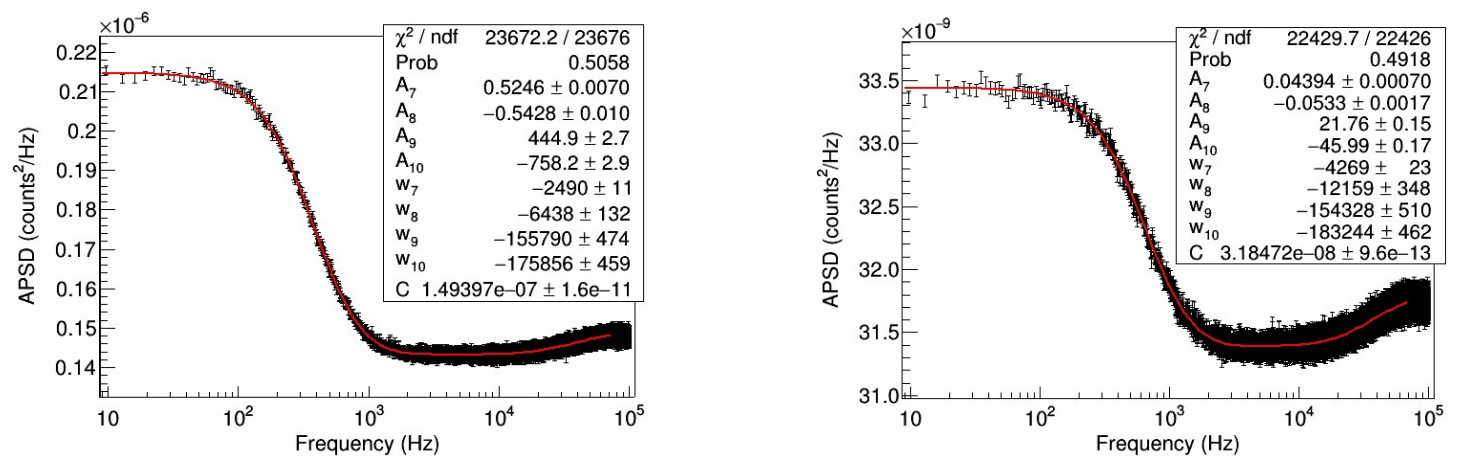

Figure 2. APSD for the Cases of 286.8 and $578.6 \mathrm{ppm}$ of Natural Boron.

The experimental data shown in Fig. 2 were least-squares fitted employing the CERN code ROOT version 6.14 running a Linux platform. The fitting function is given by Equation (1).

$$
\operatorname{APSD}(\omega)=\frac{A_{7}}{\omega^{2}+\omega_{7}{ }^{2}}+\frac{A_{8}}{\omega^{2}+\omega_{8}^{2}}+\frac{A_{9}}{\omega^{2}+\omega_{9}^{2}}+\frac{A_{10}}{\omega^{2}+\omega_{10}{ }^{2}}+C .
$$

The subscript of the coefficients $A_{j}$ and the roots $\omega_{j}$ in Figure 2 and Equation (1) are numbered starting from 7 because a six-group model for the delayed neutron families has been adopted. It is also implicit that the measurements considered frequencies $(\omega)$ much higher than $\lambda_{j}$, where $\lambda_{j}$ is the decay constant of family $j$ so that the short-lived precursors are not taken into consideration here.

Each term of the Equation (1) containing a root $\omega_{j}$ is called a mode. Several attempts have been made employing Equation (1) in order to find the best approach to fit the experimental data. Particularly, the fitted results were sensitive to their initial guess. Table 1 summarizes the final findings for the $286.8 \mathrm{ppm}$ of natural boron. Table 1 shows the maximum frequency that satisfy the criteria of having the reduced $\chi^{2}$ as close as possible to one and the probability of the $\chi^{2}$ (Prob) as close as possible to $50 \%$. The analyses were made considering sequentially one through four modes. The one mode model considers just the first term of Equation (1) and the constant $C$. This is the classical APSD behavior predicted by the point kinetic theory. For this case, the maximum frequency that this model can satisfactory fit the experimental data is $1,175 \mathrm{~Hz}$ which is well below the maximum frequency of measured data. The two-modes that follows represents the reflector-core model as firstly proposed by Cohn [9] and improved by Spriggs [3]. 
The maximum frequency reached for this model is $9,650 \mathrm{~Hz}$. The three-mode model also can not fit the complete set of measured data. Only, the four-mode model satisfactorily fitted the measured data. The maximum frequency in this case is $75.8 \mathrm{kHz}$ which incorporates the physical events at high frequencies. Another very important characteristic of the four-mode least square fitted results compared to the previous cases was the significant reduction of the fitted parameter uncertainties.

Fig. 2 shows that there are two distinct sets of the adjusted roots $\left(\omega_{j}\right)$. Two roots $\left(\omega_{7}\right.$, and $\left.\omega_{8}\right)$ at lower frequencies and two roots $\left(\omega_{9}\right.$, and $\left.\omega_{10}\right)$ at higher frequencies. It will be shown shortly in the two-group two-region model developed in this work that the first two modes of the APSD given by Eq. (1) represents the effects of thermal neutrons and the last two models those of fast neutrons. Furthermore, the similarity of the roots $\left(\omega_{9}\right.$, and $\left.\omega_{10}\right)$ for both boron diluted cases is very noticeable. This is due to the low boron concentrations employed in this work. The presence of the boric acid does not change appreciably the neutron spectra in the fast neutron energy range. Finally, the coefficients $A_{7}$ and $A_{8}$, considering their uncertainty ranges $(3-\sigma)$, are anti-symmetric $\left(A_{7}=-A_{8}\right)$. This condition is best satisfied for the $286.8 \mathrm{ppm}$ case. This feature of the fitted data will be an important finding to identify the first two modes as the kinetic behavior of the thermal group in the developed two-group two-region model kinetic model.

Due to the very large amount of experimental data, only the experimental results of the 286.6 case are shown here. All tables and figures from this point refer to this boron dilution.

Table I. Maximum achievable frequency versus number of modes for the $286.6 \mathrm{ppm}$ case.

\begin{tabular}{|c|c|c|c|}
\hline Number of Modes & Maximum Frequency $(\mathrm{Hz})$ & Reduced $\chi^{2}$ & Prob. (\%) \\
\hline 1 & 1,175 & 0.9956 & 51.36 \\
\hline 2 & 9,650 & 0.9982 & 52.39 \\
\hline 3 & 27,000 & 0.9999 & 50.02 \\
\hline 4 & 75,800 & 0.9998 & 50.58 \\
\hline
\end{tabular}

Fig. 3 shows the partial contributions of the individual modes to the measured APSD.

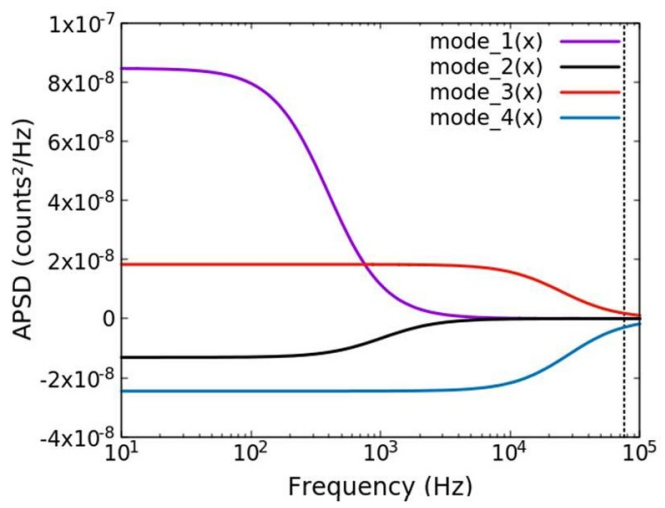

Figure 3. Partial Mode Contributions for the Total APSD for the $286.6 \mathrm{ppm}$ case.

Fig. 3 shows that the contributions of modes 3 and 4 for frequencies below $10 \mathrm{kHz}$ are approximately constants, with opposite signs and nearly cancel each other. On the other hand, for frequencies higher than around $7 \mathrm{kHz}$ the contributions of modes 1 and 2 nearly goes to zero. Consequently, modes 1 and 2 dominates the measured APSD at frequencies lower than $10 \mathrm{kHz}$ and conversely modes 3 and 4 
dominates at higher frequencies. Also, it can be concluded, at least in the first glance, that the modes 1 and 2 and modes 3 and 4 are uncoupled. These findings will give rise to the development of the twogroup two-region kinetic model for the interpretation of the measured APSD.

\section{THE TWO-GROUP AND TWO-REGION NEUTRON KINETIC MODEL}

Consider that a reactor in a critical state is suddenly perturbed and the physical constants of the transport equation are changed by a specific amount e.g., $\sigma=\sigma_{0}+\Delta \sigma$ in the case of total cross section, and a neutron source is suddenly inserted into the system. The temperature feedback is neglected for this transient. Consider further the traditional way to get the time behavior of the transient as in Ref. [10], and a two-region problem consisted of a core and a reflector region.

The kinetic equations will be derived in a two-group model. Following the traditional way, in each group, the direct transport equation is multiplied by the adjoint flux, the adjoint transport equation is multiplied by the direct neutron flux, the resultant equations are subtracted. Next, the final set of equations are integrated in the phase space $(\vec{r}, \vec{\Omega}, E)$, where $\vec{r}$ represents the neutron flux position, $\vec{\Omega}$ the neutron direction, and $E$ the neutron energy in each energy group and in each region (core or reflector). In this last step, the neutron flux is factored in an amplitude and a shape factor. The final set of equations is:

$$
\begin{gathered}
\frac{d N_{c}^{F}}{d t}=-\frac{\beta_{e f f}^{F F}}{\Lambda_{c}^{F}} N_{c}^{F}-\frac{j_{c \rightarrow r}^{F}}{\Lambda_{c}^{F}} N_{c}^{F}+\frac{j_{r \rightarrow c}^{F}}{\Lambda_{c}^{F}} N_{r}^{F}-\frac{X_{1 c}^{F T}}{\Lambda_{c}^{F}} N_{c}^{F}+\frac{F_{1 p}^{T F}}{\Lambda_{c}^{F}} N_{c}^{T}+\sum_{j} \lambda_{j} c_{j}^{F}+S_{c}^{F} \\
\frac{d c_{j}^{F}}{d t}=\frac{\beta_{j}^{F F}}{\Lambda_{c}^{F}} N_{c}^{F}+\frac{\beta_{j}^{T F}}{\Lambda_{c}^{F}} N_{c}^{T}-\lambda_{j} c_{j}^{F} \\
\frac{d N_{c}^{T}}{d t}=\frac{\rho_{c}^{T T}-\beta_{e f f}^{T F}}{\Lambda_{c}^{T}} N_{c}^{T}-\frac{j_{c \rightarrow r}^{T}}{\Lambda_{c}^{T}} N_{c}^{T}+\frac{j_{r \rightarrow c}^{T}}{\Lambda_{c}^{T}} N_{r}^{T}+\frac{X_{1 c}^{F T}}{\Lambda_{c}^{T}} N_{c}^{F}-\frac{F_{1 p}^{T F}}{\Lambda_{c}^{T}} N_{c}^{T} \\
\frac{d N_{r}^{F}}{d t}=\frac{j_{c \rightarrow r}^{F}}{\Lambda_{r}^{F}} N_{c}^{F}-\frac{j_{r \rightarrow c}^{F}}{\Lambda_{r}^{F}} N_{r}^{F}-\frac{X_{1 r}^{F T}}{\Lambda_{r}^{F}} N_{r}^{F}+S_{r}^{F} \\
\frac{d N_{r}^{T}}{d t}=\frac{\rho_{r}^{T T}}{\Lambda_{r}^{T}} N_{r}^{T}+\frac{j_{c \rightarrow r}^{T}}{\Lambda_{r}^{T}} N_{c}^{T}-\frac{j_{r \rightarrow c}^{T}}{\Lambda_{r}^{T}} N_{r}^{T}+\frac{X_{1 r}^{F T}}{\Lambda_{r}^{T}} N_{r}^{F}
\end{gathered}
$$

where $N$ is the amplitude factor, $c$ is the adjoint weighted precursor density, $S^{F}$ is the external adjoint weighted source (in the core is the intrinsic source and in the reflector is the Am-Be source), $\lambda$ is the precursor decay constant, $\beta$ is the effective delayed neutron fraction either total or in the family $j, \rho$ is the reactivity, $j_{c \rightarrow r}$ is the neutron current from the core to the reflector, $j_{r \rightarrow c}$ is the neutron current from the reflector to the core, the superscripts $F$ or $T$ refer to the fast and thermal group, respectively, the subscript $c, r$, or $j$ refer to the core, reflector, and delayed neutron family $j$, respectively. The superscripts $F F, F T$, or $T F$ represent the range where the integrals of the incident and emergent neutron energy are being performed. $X^{F T}$ represents the transfer of fast neutrons to the thermal group, $F^{T F}$ represents the production of prompt fast neutrons due to thermal fissions. $\Lambda$ is given by its traditional definition as in Ref. 10, but only in the core region it has a physical meaning.

Equations (2) through (6) represents a linear set of coupled equations. The coupling between the fast and thermal groups arises from the terms $X^{F T}$ and $F^{F T}$; which are respectively the transfer of fast neutrons to thermal group and the production of prompt fast neutrons due to thermal fissions. Some approximations have already been made in this set of equations. The reactivity in the fast group inserted by addition of boric acid in the moderator and in the reflector water has been neglected due to its lower concentration. Only the reactivity in the thermal group is considered here. 
Some details must be noted in the coupled linear set of equations (2) through (6). The reactivity in the thermal group has been split into a core $\left(\rho_{c}^{T T}\right)$ and a reflector $\left(\rho_{r}^{T T}\right)$ contributions. The final reactivity is a sum of these two contributions. The effective delayed neutron fraction was also split into a fast and a thermal part.

\subsection{Reflected Core Equations in the Thermal Group}

According to the conclusions reached in section (2), the kinetics equations for the fast and thermal groups are assumed to be uncoupled. Consequently, the set of equations for the thermal group is given by:

$$
\begin{aligned}
& \frac{d N_{c}^{T}}{d t}=\frac{\rho_{c}^{T T}-\beta_{e f f}^{T F}}{\Lambda_{c}^{T}} N_{c}^{T}-\frac{j_{c \rightarrow r}^{T}}{\Lambda_{c}^{T}} N_{c}^{T}+\frac{j_{r \rightarrow c}^{T}}{\Lambda_{c}^{T}} N_{r}^{T} \\
& \frac{d N_{r}^{T}}{d t}=\frac{\rho_{r}^{T T}}{\Lambda_{r}^{T}} N_{r}^{T}+\frac{j_{c \rightarrow r}^{T}}{\Lambda_{r}^{T}} N_{c}^{T}-\frac{j_{r \rightarrow c}^{T}}{\Lambda_{r}^{T}} N_{r}^{T}
\end{aligned}
$$

Following the traditional way of solving the kinetic equations, first it is considered a Laplace transform of Equations (7) and (8) and next its inverse transform is taken into consideration. The APSD in the reflector region is the Fourier transform of the time dependent solution arisen from the previous step and it is given by:

where

$$
\operatorname{APSD}(\omega)=\frac{A_{r}}{\alpha_{r}^{2}+\omega^{2}}+\sum_{j} \frac{A_{j}}{\omega_{j}^{2}+\omega^{2}}
$$

$$
\begin{gathered}
A_{r}=-2 \alpha_{r}\left[N_{r 0}^{T}-\frac{j_{c \rightarrow r}^{T}}{\Lambda_{r}^{T}} \sum_{j} \frac{N_{c 0}^{T}\left(\alpha_{c} \Lambda_{c}^{T}-\frac{\beta_{r}}{\alpha_{r}}\right)}{\omega_{j}\left(\omega_{j}-\alpha_{r}\right)\left[\Lambda_{c}^{T}+\frac{\beta_{r}}{\left(\omega_{j}-\alpha_{r}\right)^{2}}\right]}\right], \\
A_{j}=-2 \frac{j_{c \rightarrow r}^{T}}{\Lambda_{r}^{T}} \frac{N_{c 0}^{T}\left(\alpha_{c} \Lambda_{c}^{T}-\frac{\beta_{r}}{\alpha_{r}}\right)}{\left(\omega_{j}-\alpha_{r}\right)\left[\Lambda_{c}^{T}+\frac{\beta_{r}}{\left(\omega_{j}-\alpha_{r}\right)^{2}}\right]} .
\end{gathered}
$$

$N_{r 0}^{T}$ and $N_{c 0}^{T}$ are the initial condition for the reflector and core neutron amplitudes, $\alpha_{c}=\frac{\rho_{c}^{T T}-\beta_{e f f}^{T F}-j_{c \rightarrow r}^{T}}{\Lambda_{c}^{T}}$, $\alpha_{r}=\frac{\rho_{r}^{T T}-j_{r \rightarrow c}^{T}}{\Lambda_{r}^{T}}, \beta_{r}=\frac{j_{c \rightarrow r}^{T} j_{r \rightarrow c}^{T}}{\Lambda_{r}^{T}}$, and $\omega_{j}$ 's are the roots (either $w_{7}$ or $w_{8}$ ) of the reflected core Inhour equation which is given by:

$$
\rho_{c}^{T T}=\omega_{j} \Lambda_{c}^{T}+j_{c \rightarrow r}^{T}-\frac{\beta_{r}}{\omega_{j}-\alpha_{r}}+\beta_{e f f}^{T F} .
$$

It can be demonstrated that $A_{r}=0$, and $A_{7}=-A_{8}$, i.e., the coefficients that multiply $1 /\left(\omega_{j}^{2}+\omega^{2}\right)$ are anti-symmetric. This is the main reason to associate to the thermal group to the first two modes of the fitted measured APSD in a four-mode model.

\subsection{The Determination of $\alpha_{c}, \alpha_{r}, \beta_{r}$, and $\Lambda_{c}^{T}$}

No attempt was made to employ least square technique to infer the $\alpha_{c}, \alpha_{r}, \beta_{r}$, and $\Lambda_{c}^{T}$ values. The degeneracy among these variables is quite severe in Equation (9). The procedure adopted here starts attributing acceptable ranges for the values for these variables. Next, the $\alpha_{c}, \alpha_{r}, \beta_{r}$, and $\Lambda_{c}^{T}$ values are varied in a stepwise way from the lowest to the highest acceptable values. $\alpha_{c}$ was the outermost loop 
while $\Lambda_{c}^{T}$ was the innermost. The set of $\alpha_{c}, \alpha_{r}, \beta_{r}$, and $\Lambda_{c}^{T}$ values obtained in every step of this procedure were tested if it satisfies the conditions of having $A_{r}=0, A_{7} /\left(-A_{8}\right)=0.966$ (from the fitted values of Fig. 2), and $\rho_{c}^{T T}\left(\omega_{7}\right)=\rho_{c}^{T T}\left(\omega_{8}\right)$ from Equation (12) inside of a specific accuracy. If so, the set of $\alpha_{c}, \alpha_{r}$, $\beta_{r}$, and $\Lambda_{c}^{T}$ values are counted as acceptable and conversely it is neglected. This procedure was repeated several times and for every time the acceptable range for the variables and the final acceptable accuracy were narrower until a desirable solution was attained. Fig. 4 shows the final solution.
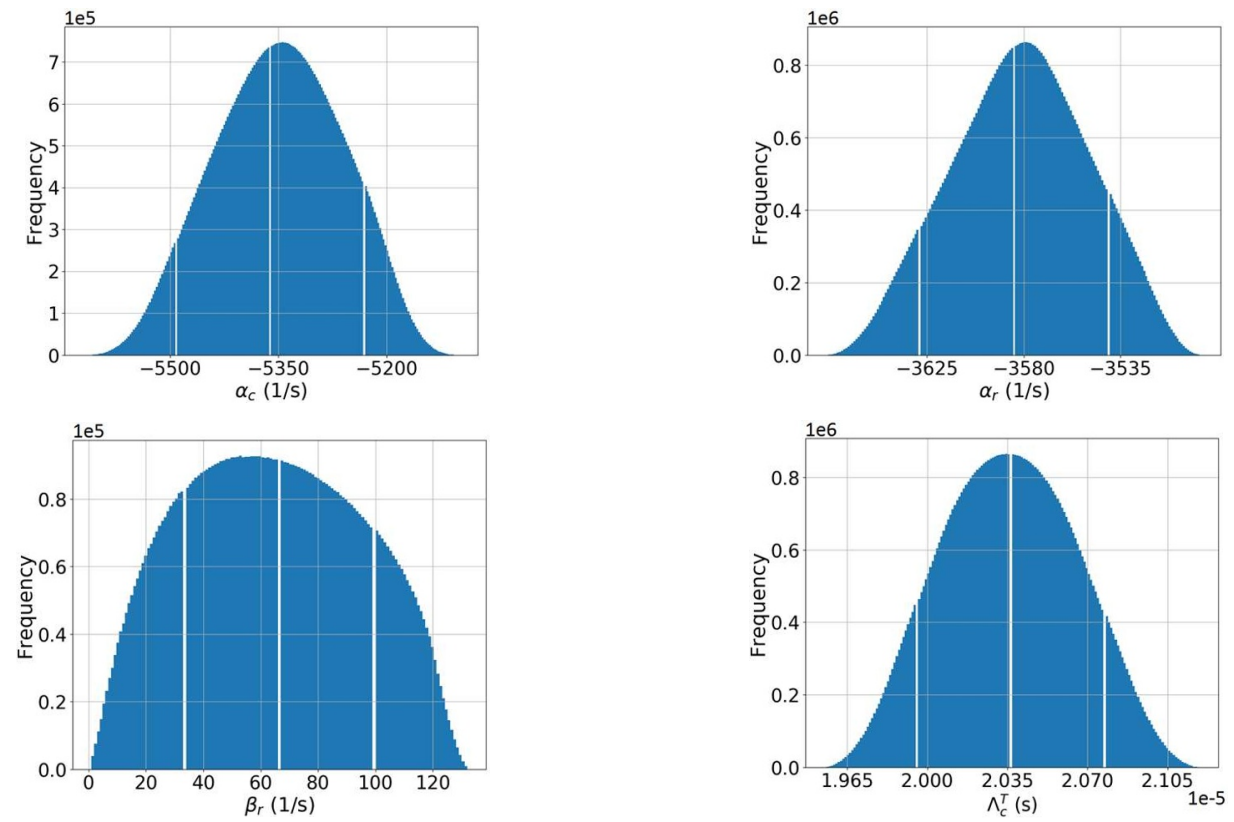

Figure 4. Frequency of Occurrence of Acceptable Values for $\alpha_{c}, \alpha_{r}, \beta_{r}$, and $\Lambda_{c}^{T}$ Against their Values for the $286.6 \mathrm{ppm}$ case.

Fig. 4 shows the frequency of occurrence of the variable set that satisfied the acceptance criteria against their value. The maximum of this curve is the most probable solution for the variable under consideration.

The uncertainties were determined by the least squares code ROOT and the APSD experimental data. The fitted curve was given by Equation (1) but now $\omega_{9}, \omega_{10}, A_{7}, A_{8}, A_{9}$, and $A_{10}$ were kept fixed and the roots $\omega_{7}$ and $\omega_{8}$ were given by:

$$
\omega_{j}=\frac{\alpha_{c}+\alpha_{r}}{2} \pm \sqrt{\left(\frac{\alpha_{c}+\alpha_{r}}{2}\right)^{2}-\alpha_{c} \alpha_{r}+\frac{\beta_{r}}{\Lambda_{c}^{T}}}, \mathrm{j} \text { equal to } 7 \text { or } 8
$$

The positive and negative signs in Equation 13 refer, respectively to $\omega_{7}$, and $\omega_{8}$. The least squares procedure with the most probable values of $\alpha_{c}, \alpha_{r}, \beta_{r}$, and $\Lambda_{c}^{T}$ as initial guesses converged satisfactorily in a single iteration and practically did not change their values, thus demonstrating that the adopted procedure was successful and accurate. Table II shows the final values along with their uncertainties.

Table II. Final values of $\alpha_{c}, \alpha_{r}, \beta_{r}$, and $\Lambda_{c}^{T}$ for the $286.6 \mathrm{ppm}$ case.

\begin{tabular}{|c|c|c|c|}
\hline$\alpha_{c}\left(s^{-1}\right)$ & $\alpha_{r}\left(s^{-1}\right)$ & $\beta_{r}\left(s^{-1}\right)$ & $\Lambda_{c}^{T}(\mu \mathrm{s})$ \\
\hline$-5,348 \pm 54$ & $-3,581 \pm 40$ & $63.4 \pm 1.9$ & $20.35 \pm 0.59$ \\
\hline
\end{tabular}




\section{CONCLUSIONS}

The experiment for the APSD measurements at high frequencies has been successfully performed at the IPEN/MB-01 reactor. The experiment was a very challenging one because it required a long acquisition times and it was very sensitive to the electronic setup. The analyses reveal that the experimental data were best fitted by a four-mode model. It was demonstrated that there were two distinct frequency regions. The first two modes dominate the lower frequency region while the last two dominates the high one and could be considered uncoupled. A two-region two-group kinetic model as developed in this work and from that it could be demonstrated that the first two modes corresponds to the thermal group and the last two ones to the fast group. Only the thermal group was analyzed in this work. The parameters inferred from the experimental data were $\alpha_{c}, \alpha_{r}, \beta_{r}$, and $\Lambda_{c}^{T}$, and were obtained with a high degree of accuracy. The most important parameter obtained so far is $\Lambda_{c}^{T}$. Neither of the available computer codes calculates $\Lambda_{c}^{T}$ directly. $\Lambda_{c}^{T}$ can of course be determined from the direct and adjoint fluxes (if available) in conjunction to its nuclear data library. The importance of this reactor physics parameter besides of being an important kinetic data is to serve as a benchmark quantity to check the magnitude of ${ }^{235} \mathrm{U} v \sigma_{f}$ data in the thermal group. $\Lambda_{c}^{T}$ is inversely proportional to this quantity and in the case of IPEN/MB-01 reactor only ${ }^{235} \mathrm{U}$ fissions in this group. The experiment analyses are still in progress and it is expected to get some other important reactor physics parameters from the experimental data such as the subcritical reactivity.

\section{ACKNOWLEDGMENTS}

The authors are grateful to Fundação de Amparo à Pesquisa do Estado de São Paulo (FAPESP) for providing the financial supports to make the IPEN/MB-01 correlator feasible under research contracts 03/01261-0 and 2013/14908-4. .

\section{REFERENCES}

1. G.D. Spriggs, "The Reactor Noise Threshold," Nucl. Sci. Eng., 116, pp. 67-72 (1994).

2. J.S. Bendat and A.G. Piersol, Random Data - Analysis and Measurement Procedures, Second Edition (Revised and Expanded), John Wiley, New York (1986).

3. G.D. Spriggs et al., "Two-Region Kinetic Model for Reflected Reactors," Ann. Nucl. Energy, 24(3), pp. 205-250 (1997).

4. R.Y.R. Kuramoto et al., "Absolute Measurement of $\beta_{\text {eff }}$ based on Rossi- $\alpha$ experiments and the TwoRegion Model in the IPEN/MB-01 Research Reactor," Nucl. Sci. Eng., 158(3), pp. 272-283 (2008).

5. E. Gonnelli, and A. Dos Santos, "The Determination of Reflector Kinetics Parameters Through Rossi$\alpha$ Experiments in the IPEN/MB-01 Reactor," Proceedings of PHYSOR 2016, Sun Valley Resort, Sun Valley, Idaho, USA, May $1-5,2016$.

6. A. Dos Santos, et. al., 2014. LEU-COMP-THERM-077: Critical Loading Configurations of the IPEN/MB-01 Reactor. In: Briggs, J. Blair (Ed.), in: International Handbook of Evaluated Criticality Safety Benchmark Experiments. NEA/NSC/DOC (95)03/I, Paris.

7. Y. Kitamura, M. Matoba, T. Misawa, H. Unesaki, and S. Shiroya, "Reactor Noise Experiments by Using Acquisition System for Time Series Data of Pulse Train," J. Nucl. Sci. Technol., 36(8), pp. 653-660 (1999).

8. J.S. Bendat, and A.G. Piersol, Random Data: Analysis and Measurement Procedures, $3^{\text {rd }}$ ed., John Wiley \& Sons, New York, NY (2000).

9. C.E. Conh, "Reflected-Reactor Kinetics," Nucl. Sci. Eng., 13, pp. 12-17, (1962).

10. G.I. Bell and S. Glasstone, Nuclear Reactor Theory, Van Nostrand Reinhold Company, New York (1970). 